\title{
Renouveau du conte et action sociale au Brésil : des conteurs bénévoles en dialogue avec leur public en situation de handicap
}

Revival of Storytelling and Social Action in Brazil: Volunteer Storytellers in Dialogue with their Disabled Public

Renovação do conto e ação social no Brasil: os contadores voluntários brasileiros em diálogo com o público com paralisia cerebral

\section{Rossane Lemos}

\section{OpenEdition \\ Journals}

Édition électronique

URL : https://journals.openedition.org/clo/6046

DOI : $10.4000 /$ clo.6046

ISSN : 2266-1816

\section{Éditeur}

INALCO

\section{Édition imprimée}

Date de publication : 18 juin 2019

Pagination : 185-212

ISBN : 978-2-85831-348-8

ISSN : 0396-891X

\section{Référence électronique}

Rossane Lemos, «Renouveau du conte et action sociale au Brésil : des conteurs bénévoles en dialogue avec leur public en situation de handicap », Cahiers de littérature orale [En ligne], 85 | 2019, mis en ligne le 19 juin 2020, consulté le 22 mars 2022. URL : http://journals.openedition.org/clo/6046 ; DOI : https://doi.org/10.4000/clo.6046

\section{(c) (7) (8)}

Cahiers de littérature orale est mis à disposition selon les termes de la Licence Creative Commons Attribution - Pas d'Utilisation Commerciale 4.0 International. 


\title{
Renouveau du conte et action sociale au Brésil : des conteurs bénévoles en dialogue avec leur public en situation de handicap
}

\author{
Rossane Lemos \\ Université Sorbonne Nouvelle - Paris 3 - Lacito
}

Au Brésil, face aux nombreux problèmes sociaux, politiques et économiques que rencontre le pays actuellement, se développe ce qu'on appelle le Tiers secteur, rassemblant les associations caritatives. Des organismes à but non lucratif se battent pour des objectifs sociaux en dehors des secteurs public et privé et font partie de ce mouvement social important au Brésil. Dans cet univers d'institutions axées sur l'engagement social qui rêvent d'une société plus développée et équilibrée, certaines ont décidé d'agir à travers le bénévolat narratif, comme la Casa. La littérature orale inspire des volontaires à intervenir dans différents contextes de la société urbaine brésilienne, cherchant à trouver des solutions face aux défis hypermodernes.

L'association Casa do Contador de Histórias ${ }^{1}$ de Curitiba s'inscrit dans le mouvement $\mathrm{du}$ 《 renouveau du conte », un phénomène qui s'est développé dans les zones urbaines du pays depuis les années 1990. Les conteurs d'histoires membres de cette association pratiquent la narration de contes à titre bénévole, dans le but de contribuer au développement de la conscience individuelle et par conséquent à la transformation du monde. Ils s'adressent plus précisément à des publics défavorisés qu'ils rencontrent dans des institutions partenaires. L'association propose aux conteurs des formations, une méthodologie et un

1. Bien qu'on puisse traduire ce nom en « Maison des conteurs d'histoires », nous allons le conserver en portugais, ainsi que son surnom « Casa ». 
répertoire. Contant dans un cadre ritualisé, ils recherchent une technique de narration neutre, ce qui caractérise un style particulier que nous proposons pour les besoins de l'analyse d'appeler « récit social ». Si les conteurs de la Casa eux-mêmes ne qualifient pas ainsi leur manière de conter, nous avons forgé cette expression de « récit social » afin de mettre en valeur l'engagement des conteurs bénévoles envers les publics défavorisés dans un style d'expression, non théâtralisé mais fortement ritualisé, qui est propre aux membres de l'association.

Parmi les différents publics défavorisés auxquels ils s'adressent, les conteurs de la Casa interviennent une fois par semaine dans une école spécialisée pour les jeunes atteints de paralysie cérébrale légère, modérée ou sévère. Comment ce « récit social » se présente-t-il dans ces conditions particulières ? Dans quelle mesure la narration d'histoires développée par des bénévoles de la Casa permet-elle d'instaurer, entre le conteur et son auditoire, un dialogue, au sens que Paulo Freire ${ }^{2}(1974,2011)$ donne à ce terme dans sa théorie de l'action dialogique ? Ces jeunes en situation de handicap ${ }^{3}$ moteur et/ou intellectuel peuvent-ils produire une « parole » et échanger, inspirés par les contes ?

Le public de l'école Vivian Marçal est constitué d'enfants et d'adolescents présentant une déficience physique neuromotrice associée à de multiples handicaps dus à la paralysie cérébrale. Cette dernière provoque des troubles moteurs souvent accompagnés de troubles sensoriels, perceptifs, cognitifs, de la communication et du comportement. En pratique, ils ont des difficultés pour parler, marcher, contrôler les mouvements des bras (manque de force ou rigidité des membres), ainsi souvent que des problèmes vésico-sphinctériens et des troubles respiratoires.

Après avoir présenté le contexte de narration bien particulier qui nous intéresse ici, nous verrons comment les pratiques narratives mettent en œuvre un « récit social » afin de conter face à un public de jeunes en situation de handicap,

2. Paulo Reglus Neves Freire est né en 1921 dans une région défavorisée du Nord-Est du Brésil. Philosophe et pédagogue, il a été professeur dans plusieurs universités brésiliennes et a développé des théories sur la conscience et l'éducation. Son premier livre, Pedagogia do Oprimido (paru en français en 1974 sous l'intitulé Pédagogie des opprimés), écrit lors de son exil politique au Chili en 1968, a été traduit dans une trentaine de langues.

3. Au lieu du terme «personne handicapée » défini par la loi «Pour l'égalité des droits et des chances, la participation et la citoyenneté des personnes handicapées » du 11.02.2005, nous allons garder les expressions « personne ayant un handicap » et « personne en situation de handicap » utilisées sur le terrain de recherche, ainsi que «personne atteinte de paralysie cérébrale ». 
Renouveau du conte et action sociale au Brésil : des conteurs bénévoles en dialogue avec leur public en situation de handicap

ce qui nous conduira à nous interroger sur la possibilité de dialogue qu'offre le type de narration proposé par les conteurs de la Casa à ces jeunes.

\section{Un contexte de narration bien particulier}

C'est dans le contexte de ce qu'on peut appeler le « renouveau du conte » au Brésil qu'interviennent les bénévoles de l'association Casa, dont il est nécessaire de présenter les fondements idéologiques avant d'analyser plus avant les pratiques.

\section{Le renouveau du conte au Brésil}

Le sociolinguiste brésilien Hudinilson Urbano confirme un retour des narrateurs sur la scène contemporaine : «Autour des années 1970, plusieurs pays ont été surpris par un phénomène urbain pour le moins curieux au sein d'une société essentiellement technologique : le retour des conteurs d'histoires ${ }^{4} \gg$ (Urbano, 2000, p. 17). Les indices d'un renouveau du conte sont surtout observés au Brésil depuis les années 1990. Ce renouvellement s'exprime dans la manière d'organiser la rencontre autour des histoires, dans le profil des conteurs et dans l'intérêt du public. Le « renouveau du conte » (Calame-Griaule, 1991) n'est pas un renouvellement des histoires elles-mêmes. En effet, les conteurs brésiliens utilisent un répertoire que l'on pourrait qualifier de « classique », inspiré de l'ensemble du patrimoine culturel de l'humanité, issu de l'oralité et ensuite transcrit et publié dans des recueils, notamment par des Européens. Nous considérons donc le terme de « renouveau du conte » comme un phénomène caractérisé par une nouvelle façon de raconter, développée par des narrateurs contemporains, vivant dans des sociétés urbaines et industrielles; et avant tout par une nouvelle figure du narrateur, dont les motivations sont différentes de celles du conteur traditionnel. Cette nouvelle expérience narrative inclut également un nouveau public, qui n'écoute plus en tant qu'héritier d'un savoir traditionnel, mais choisit de participer à un « cercle »d'histoires, motivé par des choix particuliers. Le terme « conteur ou narrateur d'histoires », tel qu'il est utilisé par les membres de la Casa, désigne les hommes et les femmes qui participent à un cours préparatoire, s'associent à un groupe consacré à la narration orale, préparent un répertoire d'histoires et fréquentent des institutions et des espaces publics afin de raconter des contes, avec l'objectif de développer un travail bénévole social dans un

4. Texte original : "Em torno dos anos 1970, vários países foram surpreendidos por um fenômeno urbano, no mínimo curioso, numa sociedade essencialmente tecnológica: a volta dos contadores de histórias" (ma traduction). 
contexte urbain. On appelle « cercle » une forme collective de regroupement de personnes qui ne s'établit pas en réseau, ni en communauté, mais qui est dessinée comme un cercle dans l'espace et a un caractère temporaire. C'est une rencontre de personnes, en reconnaissance mutuelle, autour de la narration d'histoires. Les conteurs l'appellent aussi "roda de contação de histórias", cercle de narration d'histoires. Le terme "roda" en portugais est applicable aussi à une conversation entre amis et à un moment de discussion sur un thème spécifique. La meilleure traduction trouvée pour le français est « cercle », en tant qu'association de personnes liées par un intérêt commun.

Ce qu'on appelle en portugais "renascimento do conto", la renaissance du conte, ou "renovação do conto", le renouveau du conte, représente un mouvement au Brésil, dans la mesure où les principaux aspects en sont : la reconnaissance par le gouvernement de l'activité narrative ; la quête de connaissances scientifiques sur l'art narratif et le métier de conteur ; la représentativité de la narration orale exprimée dans les chiffres du moteur de recherche Google ${ }^{5}$; les nouvelles plateformes numériques liées au sujet; le grand nombre d'événements consacrés à la narration ; l'accroissement de l'intérêt du grand public et la création de nouveaux groupes consacrés à l'art narratif. Le mouvement du « renouveau du conte » reçoit également un accueil positif du public. Il est favorisé par les processus d'urbanisation et d'industrialisation, par le désir de promouvoir la restauration des liens sociaux fragmentés par la réalité hypermoderne (Lipovetsky \& Charles, 2004) et par les déséquilibres de la politique et de l'économie du pays.

\section{La Casa do Contador de Histórias et ses bénévoles}

C'est dans un contexte particulier au Brésil que l'initiative de l'association Casa do Contador de Histórias a vu le jour à Curitiba, comme association à but non lucratif, en 2003. La Casa a été créée par un couple, elle psychologue et lui art-thérapeute, dans le but de rassembler les gens autour de la narration de contes. Aujourd'hui elle est gérée et soutenue par des bénévoles, qui consacrent beaucoup de temps à se former, à étudier les contes et à les mémoriser. Avant d'animer un cercle, les conteurs débutants suivent deux types de formation afin de révéler le

5. Une recherche effectuée sur le moteur Google montre que, au début de l'année 2016, il y avait 870000 résultats en portugais pour l'expression « contador de histórias », conteur d'histoires. En 2019, la même recherche identifie 10400000 liens. Pour l'expression « curso contador de histórias », atelier de conteur d'histoires, 360000 résultats apparaissaient en 2016 et 610000 en 2019. 
Renouveau du conte et action sociale au Brésil : des conteurs bénévoles en dialogue avec leur public en situation de handicap

Rossane LEMOS

conteur qui vit dans leur esprit, pour reprendre leurs propres mots. La première formation est un cours de base obligatoire, de douze heures, qui présente les notions théoriques initiales et offre des activités pratiques en vue de la narration. La deuxième est un programme de développement qui est proposé trois fois par an et est fortement conseillé, où les conteurs peuvent approfondir les techniques narratives et leur réflexion sur les enjeux des contes.

Dix ans après sa fondation, l'association s'est installée dans une maison d'environ $300 \mathrm{~m}^{2}$ située en plein centre-ville de Curitiba et décorée dans le style « ferme artisanale ». Quatre mois après l'inauguration, un incendie parti d'une maison voisine a considérablement réduit la surface des locaux, détruisant une grande partie de ce qui avait été rénové. La population de la ville, touchée par cette tragédie, a fait preuve de solidarité par des dons d'argent, de matériaux et de main d'œuvre. La rénovation a été terminée en mars 2015 et le siège à nouveau inauguré lors d'une grande fête. Ce bâtiment accueille les formations des conteurs et quelques cercles d'histoires.

L'association a été déclarée OSCIP (Organização da Sociedade Civil de Interesse Público [Organisation de la société civile d'intérêt public]) en 2007, une reconnaissance accordée par le ministère de la Justice pour l'important travail en faveur du récit oral et de la société. La Casa est aussi reconnue d'utilité publique municipale [utilidade pública municipal] à Curitiba depuis 2008.

La Casa regroupe des conteurs qui animent gratuitement des cercles de narration d'histoires. Ils se rendent systématiquement dans des institutions sociales pour raconter pendant environ une heure. Parmi les institutions partenaires on trouve des hôpitaux, des cliniques psychiatriques, des prisons, des écoles, des crèches et des asiles. L'ambition des conteurs est de partager avec leurs auditeurs le pouvoir transformateur des contes anciens, des légendes et des histoires fantastiques. L'idée est de faire vivre ce qu'ils appellent le pouvoir amoureux de la narration d'histoires à des personnes vivant dans des situations sociales précaires. Ce « pouvoir » est célébré par les narrateurs, qui citent « l'amoureusité » comme une ligne invisible resserrant la relation entre leur public et eux-mêmes. Un mot souvent entendu à la Casa est celui de amorosidade, différent $\mathrm{d}^{\prime} \ll$ amour » [amor], pour lequel on propose comme traduction le néologisme « amoureusité ». Ce concept décrit l'exercice de l'amour de l'autre, qui s'exprime dans n'importe quelle relation et est plus fort que la tendresse. Quand le conteur exerce l'amoureusité dans le contexte de la narration, il accorde de l'attention aux techniques de la performance, cherche consciemment des histoires appropriées et écoute patiemment l'auditeur, avec empathie. L'auditeur, pour sa part, prête ses oreilles au narrateur, rêve des intrigues des contes, participe aux cercles avec attention ; les deux vivent donc en pratique un pouvoir amoureux 
du récit social basé sur l'amoureusité. Selon les conteurs, la narration a la capacité de mettre ceux qui écoutent et ceux qui racontent dans une même ambiance de bien-être en toute conscience.

Il faut prendre en compte l'engagement associatif et le bénévolat qui motivent les narrateurs. Plus qu'une performance, l'action du conteur bénévole s'inscrit dans la conviction que la narration peut avoir des vertus transformatrices de la réalité. Le récit des bénévoles élargit donc l'idée de « renouveau du conte » en ajoutant une valeur et une fonction sociales transformatrices au profil du nouveau conteur. Le « récit social » est, tout d'abord, une pratique des bénévoles motivés par des préoccupations sociales, qui réunit ces conteurs et leurs publics au travers de l'engagement associatif, à partir d'une technique de narration non théâtralisée.

\section{Représentations et références des acteurs de la Casa}

L'intention des acteurs de la Casa est de former des groupes consolidés par la rencontre affective et de faire face à la dissolution des liens sociaux. Lors d'un entretien, la conteuse Gilse Gaio se demande : «Quel type de monde va-t-on laisser en héritage aux plus jeunes ? Il me faut faire de mon mieux pour aider ce lieu où tous vivent ensemble » (mars 2014). Une autre conteuse, Lídia Hanke, précise : «Nous sommes invités à changer notre réalité, celle qui fait bouger les gens comme des inconnus par rapport aux autres, alors qu'ils sont des frères qui partagent la Terre » (septembre 2014). L'envie de changer le monde par le biais de la narration se retrouve chez la majorité des conteurs de la Casa. L'ethnologue et écrivain français Michel Valière pose une question fort pertinente à propos de la fonction sociale du récit : « Les conteurs auraient-ils donc, par leur performance, la capacité "linguistique" de réarranger leur société, ou mieux, le monde ? » (2006, p. 164). «Certainement », affirment souvent les bénévoles de la Casa.

Nous avons observé ${ }^{6}$ ces conteurs inquiets des dérives de la société contemporaine qui voulaient participer à la construction de relations plus humaines, plus pacifiques et cohérentes entre les gens. Ce but n'est pas sans lien avec celui de l'educação libertadora [éducation libératrice ${ }^{7}$ ] telle qu'elle est prônée

\footnotetext{
6. Lors d'enquêtes de terrain menées entre 2014 et 2017.

7. Freire propose une éducation critique pour la transformation des individus et de la société, basée sur le dialogue et la praxis (réflexion-action). Conçue comme une pédagogie, cette proposition visait initialement les relations entre les enseignants et les élèves. Cependant, la pensée philosophique de Paulo Freire est souvent projetée sur les relations sociales, devenant interdisciplinaire. L'éducation libératrice (ou problématisante ou dialogique) est basée sur : la conciliation, l'égalité des capacités d'apprendre et d'enseigner dans des expériences collectives ; l'horizontalité des relations
} 
Renouveau du conte et action sociale au Brésil : des conteurs bénévoles en dialogue avec leur public en situation de handicap

Rossane LEMOS

par Freire $(1974,2011)$, une pédagogie-philosophie invitant à participer à la construction de relations humaines réinventées à partir de la réflexion-action et du dialogue. Avec des objectifs similaires à ceux de Freire, les conteurs défendent la nécessité d'un travail avec les individus qui forment les groupes, afin d'établir un nouveau paradigme pour l'humanité.

Edgar Morin parle d'une quête semblable et la relie à l'action individuelle :

Nous voyons donc bien la complexité, la multiplicité constitutive de l'ultime éthique : faire émerger l'humanité. Elle comporte aussi, nécessairement, l'éveil en chacun de l'humanité. [...] C'est à chacun de commencer à commencer, ne serait-ce qu'avec lui-même. (Morin, 1981, p. 86)

Les conteurs sont d'avis que des individus plus conscients sont aussi plus enclins à former des groupes solides prêts à la transformation sociale. Ils veulent donner l'occasion à tous ceux qui participent à l'expérience narrative d'approfondir leur conscience à propos de leurs défis personnels et des solutions possibles afin, ensuite, de repartir avec un espoir renouvelé et d'exercer le droit de rêver. Selon Freire, celui qui rêve, qui se pose des questions plus consciemment et qui met en place des changements est capable d' « exprimer le monde ${ }^{8} \gg$. Ainsi, on constate que la conteuse Anete Ferraz fait confiance à ce qu'elle voit se révéler dans les cercles qu'elle anime, même si elle ne le comprend pas très bien : «C'est comme si les personnes se découvraient elles-mêmes, une sorte d'auto-découverte, une connaissance approfondie de soi-même $\gg$ (avril 2014).

La base théorique de l'équipe de la Casa s'inspire des archétypes de Carl Gustav Jung, du « monomythe » de Joseph Campbell et pour une part importante des théories anthroposophiques de Rudolf Steiner. Ces trois influences ne sont pas simplement juxtaposées et adoptées en totalité ; seuls certains de leurs éléments sont introduits dans la pratique narrative de la Casa selon des choix qui lui sont propres. De cet ensemble de théories et de l'expérience

humanistes, l'espoir, le passé et l'avenir comme pont pour la construction critique du présent, la communication « avec » et non «pour » les individus, la richesse de la diversité des avis et l'union de tous pour la libération de tous. Finalement, elle veut promouvoir chez le peuple touché par une action éducative une conscience claire de sa condition objective.

8. La « pronúncia do mundo », dire/exprimer le monde, selon Freire (1974) est le droit à l'énonciation de la parole, la réflexion à propos de la réalité, l'action sur ce qui ne semble pas cohérent et le dialogue constitutif des relations sociales. 
pratique des bénévoles concernés surgit une méthodologie singulière, propre à la Casa, qui donne naissance au « récit social » et à ses caractéristiques.

\section{Des pratiques narratives au « récit social »}

Le cercle animé par les bénévoles dans la perspective du « récit social » se déroule quant à lui d'après une méthodologie et un répertoire proposés par l'association depuis sa fondation et qui doivent être respectés lors de chaque séance dans les institutions partenaires. Du fait que la Casa s'adresse à des publics défavorisés, l'association attend du conteur qu'il observe avec une rigueur particulière ses recommandations.

\section{Organisation du cercle}

Toute rencontre suit un scénario et une structure à peu près stables, le conteur étant habituellement debout (sauf quand il s'adresse aux enfants des crèches). D'habitude, un cercle compte trois conteurs, sinon au moins deux. Il est rare qu'un conteur anime un cercle seul. Chacun d'entre eux raconte une histoire, dans un ordre établi d'avance. Chaque narrateur a ses propres habitudes, mais généralement, ils se servent d'un chapeau de conteur, d'un T-shirt personnalisé de l'association, d'une bougie allumée et d'une clochette « pin », qui retentit au moins trois fois avant qu'une histoire ne commence. Les trois coups font référence à la «tripartition sociale » de l'anthroposophie, qui recommande l'équilibre entre les pensées, les sentiments et les actions. Le chapeau, de laine ou de feutre, doit nécessairement comporter trois pointes, également en relation avec l'anthroposophie. Le format du chapeau développé par la Casa est adopté par tous les volontaires. Le « pin » est une sorte de percussion à tube, composée d'une mailloche et d'une base, toutes deux en bois, et d'un fil attaché à un tube de métal qui produit une seule note (la) lorsqu'il est frappé. Les bénévoles de la Casa estiment que le «la » produit par cet instrument contribue à la permanence de l'esprit individuel dans le moment présent.

Parmi les étapes et les éléments présents dans un cercle, nous trouvons : la pratique de l'« eurythmie » pour le groupe de conteurs et pour le public, le récit de poèmes pour les conteurs et le public, l'utilisation de consignes, chansons, formulettes et devinettes, etc. L'eurythmie, l'une des expressions de l'anthroposophie, met en relation les sons des voyelles avec les gestes et mouvements du corps. Chaque voyelle correspond donc à un mouvement précis. Pour les anthroposophes, chaque son possède une essence profonde qui s'exprime dans le mouvement, rendant possible une action bénéfique aux sentiments de celui qui la pratique. Cette technique développe également la sensibilité, la 
Renouveau du conte et action sociale au Brésil : des conteurs bénévoles en dialogue avec leur public en situation de handicap

notion d'espace-temps et les capacités motrice, rythmique, auditive et sociale. L'eurythmie est utilisée dans trois domaines : l'art du spectacle, la thérapie et l'éducation.

La structure de la séance ordonne les textes des histoires afin de provoquer l'éveil, la réflexion et le calme chez les auditeurs. La séance se termine avec les commentaires du public et la «méditation du Rá ». Cette méditation est l'activité qui clôt le cercle avec un appel à la bonne chance et au succès des projets de tous ceux qui sont présents et de leurs familles. Un conteur dirige cette activité, invitant les auditeurs à frotter leurs mains au niveau de la poitrine de manière à les réchauffer, pendant qu'il leur propose de penser à leurs propres intentions et à celles des autres, de faire une demande à l'univers, une sorte de prière. Le coordinateur demande ensuite aux auditeurs d'agiter les mains et les doigts de manière à « propager » les demandes et les intentions dans l'espace. Puis, il compte jusqu’à trois et tous s'écrient « Rá ! ». Régulièrement, le public applaudit à la fin de cette activité.

Le discours de l'association sur sa méthodologie est très clair quant à la séparation entre la narration d'histoires et la performance théâtrale, même si en pratique, il semble plus difficile de l'appliquer. Cette injonction suit une tendance existant au Brésil qui a été bien retracée par la conteuse et chercheuse brésilienne Cléo Busatto, qui dissocie ainsi le théâtre de la narration :

Dans la narration orale on ne représente pas un personnage, on suggère et on laisse à l'auditeur non seulement la construction de ce personnage, mais aussi de l'espace et de l'action, en accord avec ses références internes. C'est ce que j'appelle l'art de la suggestion : la narration orale, qui est différente de l'art de la représentation : le théâtre ${ }^{9}$. (Busatto, 2007, p. 33)

Les conteurs d'histoires pratiquent ce que nous pouvons appeler un « récit performé non théâtralisé » où ils n'imitent pas les voix des animaux, ni ne portent de déguisements adaptés à un scénario. Ils cherchent un rôle neutre de narrateur et racontent une histoire dont ils «témoignent » se « dérouler » au milieu du cercle. « Trouver ce lieu de neutralité et y rester n'est pas facile », avoue le conteur bénévole Fabrizio Marangoni (janvier 2016).

9. Texte original : "Na narração oral não se representa um personagem, antes, se sugere e deixa-se ao ouvinte a construção desse personagem, do espaço e da ação, de acordo com os seus referenciais internos. É o que chamo de arte da representação - teatro; e arte da sugestão - a narração oral" (ma traduction). 
L'éducatrice et conteuse brésilienne Cristiane Velasco (2018) a écrit sur la perspective du «J'étais là-bas », où le conteur devient, avec ses yeux imaginaires, témoin des scènes du conte. Par conséquent il raconte ce qu'il a « vu » se passer au milieu du cercle, dans le « rapport de conviction (mentir vrai) » (Hernandez, 2006, p. 225). C'est une activité complexe et un vrai défi pour un narrateur débutant. Cependant, la narration sans théâtralisation est une condition pour la réalisation pleine du « récit social » à la Casa.

\section{Choix du répertoire}

La Casa propose à ses membres conteurs la « carte pour l'âme », une recommandation concernant le répertoire de référence adapté à chaque public qui est issue des recherches théoriques et des observations sur le terrain faites par les bénévoles de l'association. Les enfants et les jeunes en situation de handicap de l'école Vivian Marçal présentent d'importantes limitations pour réaliser des mouvements libres. La Casa leur propose donc des contes à la première personne pour que les auditeurs imaginent ou rêvent qu'ils en sont les héros en réalisant des prouesses physiques. Selon ce document, les fables aussi sont recommandées pour ce public, notamment celles orientées vers des expériences personnelles; les conteurs évitent les récits d'expériences collectives. Selon la Casa, les contes répétitifs et rythmés ont la capacité de resserrer le lien entre l'âme et le corps. Ce type de texte est donc également recommandé. Parmi les suggestions (quelque peu floues), nous trouvons aussi les contes de fées, avec des personnages-miroirs comme « Jeannot et Margot » des frères Grimm, les contes sur les animaux, les contes modernes qui traitent des sujets du quotidien urbain et les histoires « éclairantes », c'est-à-dire philosophiques, qui peuvent apporter un contenu spirituel. À la Casa, on pense que les récits aident ces enfants et ces jeunes à « voyager » dans leur imaginaire, avec des scénarios fantastiques et des réalités qui les libèrent pour un temps de leurs fauteuils roulants et de leurs problèmes de mobilité. Hans Christian Andersen est une référence pour les conteurs de la Casa s'adressant à ce public.

Le répertoire suggéré par l'association est construit à partir d'une hiérarchie verticale, car le responsable de sa rédaction, le département d'action sociale de la Casa, se base sur les principes de Steiner, Campbell et Jung, ainsi que sur l'expérience des différents publics, afin de proposer ses recommandations. Il est évident que le choix des histoires repose davantage sur des textes rassemblés par des écrivains européens que sur des contes inspirés de la réalité brésilienne, d'histoires, de légendes ou de mythes régionaux. Cette tendance du répertoire de la Casa est probablement le reflet de la base anthroposophique de ses théories dont les racines et influences sont européennes. 
Renouveau du conte et action sociale au Brésil : des conteurs bénévoles en dialogue avec leur public en situation de handicap

Rossane LEMOS

Les histoires racontées pour les publics de la Casa sont choisies par les conteurs individuellement et la construction de ce répertoire part de la « carte pour l'âme » et de leurs expériences personnelles.

\section{Le public de la Casa}

Les conteurs s'adressent à des publics vivant des situations de « risque social » ou de « vulnérabilité ${ }^{10}$ » et croient à un pouvoir transformateur du conte transmis par la narration orale sur ces sujets en particulier. Ils proposent des activités au grand public tous les mois et aux institutions partenaires au moins tous les quinze jours. Parmi ces institutions, en plus de l'école qui fait l'objet de cette étude, on trouve des hôpitaux qui accueillent des adultes avec des troubles psychiatriques, des centres de formation pour des enfants et jeunes démunis ; des établissements d'accueil pour personnes âgées ; et des prisons où des jeunes purgent leur peine.

Le conteur de l'association ne choisit pas son public. La distribution est faite par le département d'action sociale de la Casa en tenant compte du profil du conteur, de la disponibilité des membres des groupes ou de la mise en place des équipes dans les institutions. Ce département considère les compétences émotionnelles à développer chez les bénévoles en rapport avec un public spécifique. Par exemple lorsque la conteuse Maria da Glória Bittencourt a rejoint le programme de bénévolat, elle a demandé à l'administration de l'association de ne pas travailler à l'école Vivian Marçal. Néanmoins, la Casa lui a proposé ce public, invitant Maria da Gloria à faire face à son blocage émotionnel par rapport à l'état des auditeurs. La narratrice parle du défi qu'elle a affronté peu à peu : « Ce fut la meilleure chose qui m'est arrivée, je les adore. » Huit ans plus tard, le département d'action sociale a proposé à la conteuse un changement afin de rencontrer d'autres publics, une invitation qu'elle a volontiers acceptée.

Dans la photo ci-dessous, Maria da Glória vient de raconter son histoire aux jeunes de l'école Vivian Marçal. Elle esquisse un large sourire, ses yeux brillent et elle marche fermement et de manière spontanée hors du cercle. Interrogée sur la

10. La discussion sur les concepts qui séparent ces deux conditions - le « risque » et la «vulnérabilité »- n'est pas encore achevée au Brésil. Pour résumer ces concepts basés sur tout ce nous avons entendu des conteurs de la Casa lors des recherches, nous appellerons « risque social » la condition qui met en danger l'intégrité physique, psychique et émotionnelle de l'individu, et nommerons «vulnérabilité » la carence économico-financière dans laquelle vit l'individu, qui peut être additionnée ou non aux aspects du « risque social », c'est-à-dire, au manque des conditions minimales de développement de la vie dans le groupe. 
durée de ce sentiment de bonheur dans sa vie quotidienne, elle répond « jusqu'au prochain cercle $\gg$ (juin 2014).

Les motivations pour le « récit social » des conteurs interrogés sont multiples, mais l'envie d'agir pour transformer le monde semble être la plus importante. Il

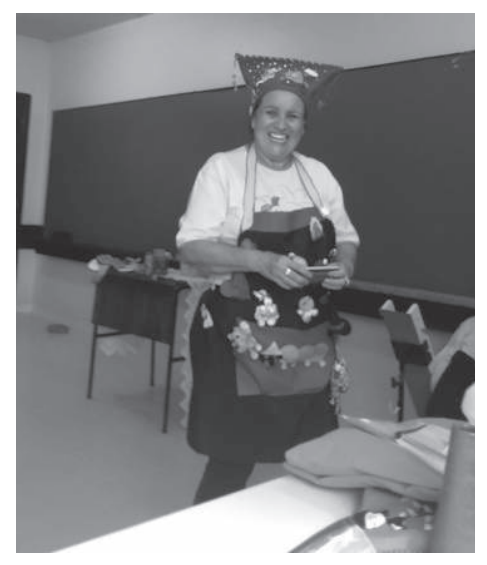

Photo 1

La conteuse Maria da Glória exprime son contentement à la fin de sa narration.

(c) Rossane Lemos, 2014

serait aisé de condamner la naïveté de cet objectif, car changer le « monde » par la narration semble un défi titanesque. Cependant, il ne s'agirait pas tant de changer le monde que d'agir sur les relations humaines entre les conteurs et leur entourage proche, avec lequel ils partagent des expériences et construisent la collectivité.

Les conteurs affirment qu'ils sont, au moins, en mesure de se changer eux-mêmes en développant une conscience critique de soi et de la société. Avant de commencer un cercle dans la même école, le conteur Renato Martins conseillait aux narrateurs débutants : « Ne croyez pas que vous allez raconter des histoires pour "sauver" ces jeunes, pour leur faire du bien. Cette occasion nous fait beaucoup plus de bien à nous conteurs qu'à eux » (mars 2014). D’après Freire, « personne ne libère autrui, personne ne se libère seul, les hommes se libèrent ensemble » (1974, p. 44). L'interaction avec les auditeurs permet aux conteurs de marcher vers un changement souhaité de leurs mondes personnels.

Les spécificités de ce public en situation de handicap nous ont conduites à mener une réflexion à propos de l'ensemble de la narration proposée par les bénévoles dans cette école, ainsi que de la participation des jeunes à cette pratique. 
Renouveau du conte et action sociale au Brésil : des conteurs bénévoles en dialogue avec leur public en situation de handicap

\section{Raconter des histoires à des jeunes en situation de handicap}

L'école d'éducation spécialisée Vivian Marçal est l'une des plus anciennes institutions partenaires de la Casa. On y reçoit les conteurs dans deux lieux : au siège principal et au siège secondaire (sede et subsede), qui accueillent environ 200 enfants et jeunes ayant des handicaps. Les cercles regroupent, à chaque fois, entre quinze et trente enfants et adolescents présentant des séquelles liées à une paralysie cérébrale. Les conséquences de cette condition sont des limitations physiques, motrices et cognitives, à différents degrés (de modéré à sévère) ainsi que des troubles de la parole. Malgré ces conditions, ces jeunes expriment des signes physiques et émotionnels lors de l'écoute d'histoires. Ces réactions pourraient-elles être considérées comme une «parole » particulière ?

\section{La «parole » des jeunes atteints de paralysie cérébrale}

Dans cette école, l'une des caractéristiques du public est sa difficulté à s'exprimer, principalement par la parole. Selon leurs professeurs, les élèves entendent ce qui est dit, le comprennent, mais, dans la plupart des cas, sont incapables d'envoyer un message clair et immédiat en retour. L'échange entre les narrateurs et les auditeurs est donc très subtil. Ceux qui sont capables de s'exprimer par la parole le font par des phrases courtes ou babillent quelques mots.

Lídia, sur la photo ci-dessous, s'adressait à l'une des auditrices qui parlait très doucement. À chaque mot de l'adolescente, la conteuse le répétait d'une voix plus forte pour que tous puissent suivre le raisonnement de la jeune fille. Elle parlait des personnages qu'elle aimerait jouer malgré son handicap visuel.

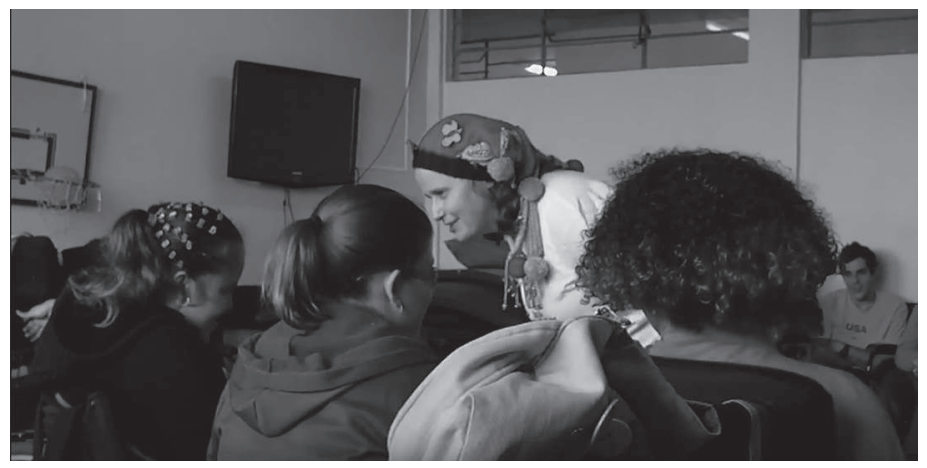

Photo 2

La conteuse Lídia amplifie la parole d'une élève de l'école Vivian Marçal.

(C) Rossane Lemos 2014 
Lors d'une séance, nous avons observé un écart par rapport au cadre proposé par la Casa, fait afin de s'adapter au public. La méditation de clôture « Rá », le cri final, qui constitue habituellement un lien spécial entre les auditeurs et les conteurs, a été remplacée. Le conteur Renato a invité les élèves à laisser voler leurs intentions dans les nuages et à souffler jusqu'à ce qu'elles gagnent le ciel, en silence. Il n'y a pas eu de « Rá », parce que la majorité des élèves présents ce jour-là n'étaient pas capables de produire le son « rá ».

Ainsi, l'association encourage les conteurs à être attentifs et à noter dans leur rapport les comportements et les réactions physiques du public pendant le cercle. Les observations portent surtout sur les signes suivants : respiration, mouvement corporel, regard, inclinaison de la tête, posture de la colonne vertébrale, niveau sonore et agitation des membres, niveau d'attention et vitalité. Selon nos enquêtes ${ }^{11}$, il est possible d'affirmer qu'il y a chez les auditeurs des changements observables entre le début et la fin d'un cercle.

$\mathrm{Si}$ nous observons les différents publics de la Casa, nous notons des changements au niveau de leurs caractéristiques physiques et émotionnelles à partir des expressions faciales, de la position de leur corps et de leur état émotionnel. Plus précisément, nous relevons une influence de la narration sur leur attention, leur disponibilité et leur participation. En général, au début du cercle, certains secouent incessamment les jambes, comme s'ils étaient anxieux ; d'autres croisent les bras. Au fur et à mesure du déroulement des histoires, ils décroisent leurs bras et leur concentration s'améliore. Dans la plupart des cas, le public réduit l'agitation des membres, relâche les muscles du thorax et prend une posture droite sur sa chaise ou, si l'histoire est romantique, certains cherchent le contact avec leur voisin s'ils se connaissent. En ce qui concerne les jeunes de l'école Vivian Marçal, les signes corporels sont plus subtils.

Avant tout, il faut noter que ces jeunes ne participent pas aux échanges de politesse existant habituellement entre l'auditoire et le narrateur puisqu'ils ne comprennent pas toujours ce qu'on attend d'eux en tant que public. Toutes les

11. Durant la première étape de la recherche, entre les mois de mars et mai 2014, nous avons pu suivre 33 cercles d'histoires dans les institutions partenaires de la Casa. Nous avons observé aussi deux cercles mensuels ouverts au grand public au siège de l'association et des cours préparatoires. Les prestations des narrateurs observés ont été enregistrées sous forme audio ou vidéo, ainsi que les entretiens individuels. Pendant les années de 2015, 2016 et le premier semestre de 2017, nous avons modifié les formes de relation (des entretiens directs selon des grilles spécifiques, des entretiens semi-directifs auprès des conteurs), par une vie commune avec les narrateurs, où de nombreuses autres expériences narratives ont été observées. 
Renouveau du conte et action sociale au Brésil : des conteurs bénévoles en dialogue avec leur public en situation de handicap

réactions sont donc spontanées, précisément à cause de l'absence de mécanismes qui provoquent des comportements répondant aux normes sociales attendues.

La conteuse Lídia a observé les enfants de l'école Vivian Marçal pendant sept ans. Sur la réaction de ce public à la narration, elle dit :

Je vois qu'après avoir entendu l'histoire ils tentent de s'exprimer. Il y a eu des histoires pendant lesquelles ils ont pleuré, ri de manière décontractée, ri nerveusement, en dehors de la normale. On parvient à distinguer cette différence. La narration stimule réellement cette «parole », cette communication. (mai 2014)

Bien entendu, ces jeunes n'ont ni les mêmes gestes ni les mêmes regards que les autres auditeurs avec lesquels la Casa travaille. Cependant, il y a toujours des réactions, une participation et des contributions du public. Lors d'un cercle que nous avons observé, l' « eurythmie » a eu lieu et trois élèves y ont participé au début sur un total de vingt. Ils se sont impliqués dans la limite de leurs possibilités physiques. À la fin, les conteurs ont proposé de refaire l'eurythmie. La participation à l'activité a été plus expressive et cette fois-ci neuf jeunes y ont participé. On voit que durant le cercle non seulement la motivation des jeunes à participer à l'activité a augmenté, mais aussi que leur condition physique s'est améliorée de façon à permettre qu'ils bougent pour réaliser l' « eurythmie » dans leur limite.

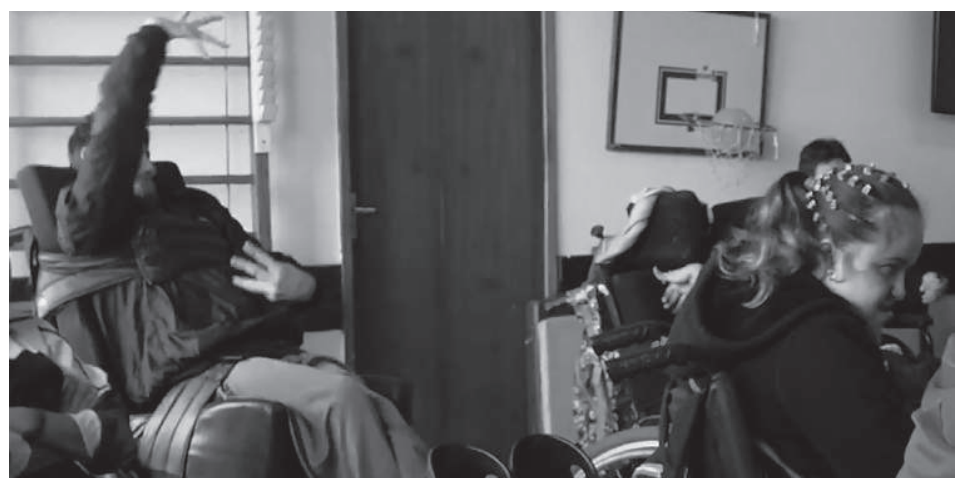

Photo 3

Un jeune bouge les mains et fait des efforts afin de participer à l'activité proposée.

(c) Rossane Lemos, 2014

Pendant un autre cercle, à la fin de la dernière histoire, quelques élèves qui paraissaient distraits ont tout de même pu nommer la couleur de l'étoile (le personnage du conte) et donner leur avis sur l'histoire. Un jeune garçon atteint 
de paralysie cérébrale modérée a formé cette phrase complète en balbutiant : « J'ai aimé l'histoire de l'étoile verte » (mars 2014). Nous voyons ici un exemple de réaction au niveau de l'attention de ce public.

\section{Des expériences particulières}

D'habitude, l'ambiance des cercles dans cette institution est assez agitée pour diverses raisons, en particulier le fait que le personnel soignant emmène et ramène les enfants pour les changer et leur administrer des médicaments. Un après-midi, le cercle a démarré dans une salle centrale qui est un lieu de passage vers d'autres espaces de l'école. Les élèves étaient déjà réunis pour écouter des histoires, quand un enfant est arrivé en fauteuil roulant. Il émettait de forts gémissements et avait d'énormes difficultés pour respirer. Ce garçon avait besoin d'un appareil respiratoire et cette machine rend chaque inspiration et expiration très bruyante. Ces conditions ont été de vrais défis pour la concentration des trois narrateurs bénévoles. Mais, comme ils l'ont assuré eux-mêmes, ils sont là justement pour raconter à ceux qui ont besoin des histoires afin de faire face à la souffrance. L'enfant était agité et tous les participants l'étaient également, du fait de ce bruit permanent qui les dérangeait. Mais au fur et à mesure que les histoires étaient racontées, la respiration de l'élève se calmait. Peu à peu la machine produisait moins de bruit et la respiration de l'enfant devenait quasiment imperceptible, au point de ne plus déconcentrer ses camarades. Il respirait au même rythme que les autres enfants. Les conteurs bénévoles attribuent ce changement d'état de santé de l'enfant aux vertus transformatrices du conte et de la narration, en tant que pouvoir amoureux. Selon eux, le bien-être et la conscience seraient plus accessibles après l'écoute d'une histoire.

Lídia parle des expériences qu'elle a vécues avec les histoires et ne doute pas du pouvoir transformateur de celles-ci :

Les histoires transmettent des images qui nous renvoient à des découvertes très importantes pour nous, chacun d'entre nous, à chaque moment, elles nous aident à trouver notre chemin. Elles m'ont aidée à trouver mon chemin lorsque j'en ai eu besoin. L'histoire d' « Hamed et la princesse intrépide ${ }^{12}$ » est un bon exemple. J'ai beaucoup travaillé avec cette histoire. Je l'ai donc étudiée, dessinée, j'ai fabriqué les personnages avec de la laine et cette histoire m'a aidée à découvrir des vérités que je ne voyais

12. CAMPBell, 1952, p. 16-23. 
Renouveau du conte et action sociale au Brésil : des conteurs bénévoles en dialogue avec leur public en situation de handicap

pas dans ma vie. C'est là que les histoires démontrent leurs vertus thérapeutiques en aidant l'auditeur à se découvrir. (mai 2014)

Les fondements de ces vertus thérapeutiques ne sont pas interrogés par ces bénévoles, qui trouvent suffisant le fait de témoigner des changements sur eux-mêmes et, pendant les cercles, sur le public.

L'une des situations les plus intéressantes à cet égard s'est déroulée un matin dans la salle principale du siège secondaire de l'école. Le cercle a commencé et une fille était allongée sur un matelas au sol le long du cercle ; une adolescente de douze ans, pâle et présentant de graves troubles cognitifs. Elle ne pouvait pas bouger en raison de déficiences physiques dues à une paralysie cérébrale sévère. Elle avait le regard fixé sur le plafond, était apathique et semblait totalement absente du cercle. Deux histoires ont été racontées et, au début de la troisième, elle a commencé à réagir en clignant des yeux, en bougeant les bras et en regardant ses mains. Elle semblait avoir pris conscience de son corps, de l'ambiance et du moment présent. À la fin de l'histoire elle a perçu notre présence, assise à trois mètres d'elle, et elle nous a regardée intensément. Cet échange de regards a suscité notre réflexion sur les modes d'interaction et de dialogue après l'écoute d'une histoire.

La séquence de photos ci-dessous montre un autre exemple de réaction des jeunes à la narration. Dans la première photo, au début de l'histoire, pratiquement tous les adolescents regardent ailleurs que dans la direction de la narratrice. Sur la deuxième image, au cours de la narration, le premier élève à gauche regarde et sourit à la narratrice ; le deuxième lève la tête sur le côté, comme pour rechercher celle qui raconte. Sur la troisième photo, presque à la fin de l'histoire, ce deuxième élève regarde également la narratrice, bâille et sourit. 

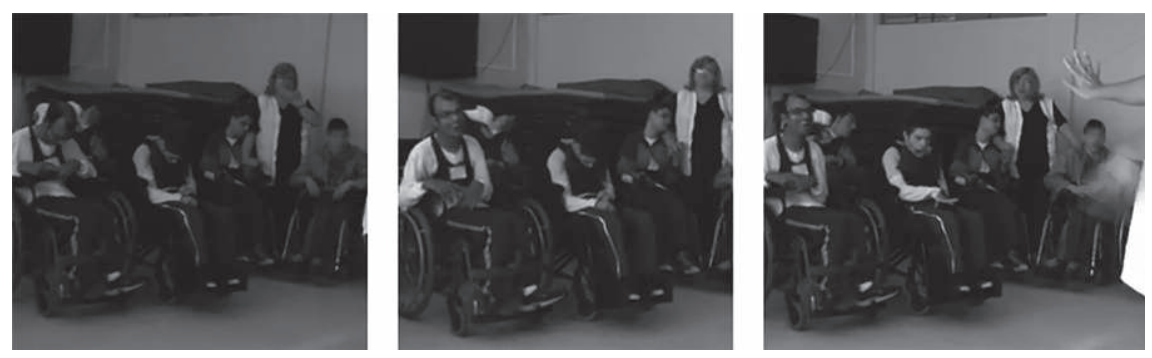

Photos 4, 5, 6

Séquence de photos prises lors de la narration d'une histoire à des jeunes atteints de paralysie cérébrale montrant leurs changements de postures corporelles.

(c) Rossane Lemos, 2014.

Cette séquence de photos présentant les réactions des élèves peut être comprise comme une forme de leur participation au cercle. Nous pouvons penser qu'il s'agit de leur façon de dialoguer avec les conteurs et les autres adolescents et, pourquoi pas, avec le conte.

Selon les observations des responsables de l'école Vivian Marçal, la narration améliore l'affectivité et l'expression des émotions. Après un cercle les élèves retournent dans leurs salles «plus concentrés, enthousiastes et avec une posture corporelle plus équilibrée ${ }^{13} \gg$. Cependant, les aspects culturels et émotionnels sont les bénéfices les plus importants du travail des conteurs bénévoles, selon les responsables de l'école. Ils invoquent le fait que les élèves n'ont pas accès à des activités culturelles ou artistiques du fait de leur mobilité réduite. Le cercle d'histoires est ainsi l'occasion de s'ouvrir au monde, en plus d'encourager leur intérêt pour la littérature. Ils constatent l'enrichissement de l'action pédagogique au sein de l'école grâce à la narration systématique.

Les transformations comportementales et corporelles après l'écoute d'une histoire narrée par les bénévoles semblent donc reconnues et enrichissent l'expérience des bénévoles et des auditeurs. Finalement, les interfaces sociales de l'engagement associatif dans un cadre ritualisé, le pouvoir amoureux de la

13. Rapport annuel rédigé par les responsables des institutions, en réponse au travail bénévole narratif des conteurs de la Casa. Archives 2013 imprimées de la Casa. 
Renouveau du conte et action sociale au Brésil : des conteurs bénévoles en dialogue avec leur public en situation de handicap

narration d'histoires et la réception de ces contes par le public en situation de handicap nous invitent à penser à une dimension de « dialogue » possible entre le narrateur et ce public spécifique. Interrogeons cette dimension dialogique afin de mieux comprendre la pratique du « récit social » telle qu’elle est proposée par les conteurs de la Casa.

\section{Quel dialogue possible?}

Quand nous considérons ce que dit la conteuse Lídia : « La narration stimule réellement cette "parole", cette communication » des jeunes atteints de paralysie cérébrale, nous pensons que la place attribuée au dialogue inclut une perspective interactionniste à laquelle tous contribuent de manière subjective, au-delà de la parole. Bien que les narrateurs ne citent pas Paulo Freire parmi leurs références, nous avons mis en relation leur pratique $\mathrm{du}$ 《récit social » avec la notion freirienne de dialogue. Notre décision a été motivée par l'écoute du discours des bénévoles sur leur pratique, quand ils emploient des mots clés tels que « amour », « foi » et « humilité », notions présentes dans la théorie du dialogue de Freire.

Dans son ouvrage Pédagogie des opprimés (1974), Freire affirme que le dialogue ne favorise pas seulement la transformation, mais qu'il est la transformation. Par le dialogue, il croit possible d'expérimenter un développement individuel et une transformation des relations sociales. Dans quelle mesure un dialogue entre narrateurs et public présentant de tels handicaps est-il possible?

D’après Freire, pour créer les conditions du dialogue, les personnes concernées doivent faire l'expérience de l'amour, de la foi, de l'humilité, de la confiance, de l'espoir et de la conscience critique. Afin de résumer ces éléments indispensables au dialogue selon l'auteur, il faut dire que l'amour est une action engagée avec le monde et avec l'autre, à partir de la reconnaissance mutuelle. L'action remplie d'amour est centrée sur la libération de toutes les personnes de ceux qui les oppriment. La foi est une conviction que les gens participent à des processus pour se transformer et transformer le monde. C'est également la conviction de « plusêtre $^{14} \gg$, c'est-à-dire la confiance dans le développement permanent de l'être humain. L'humilité est un principe selon lequel tous les individus ont quelque

14. Le ser mais [plus-être] est considéré comme un droit, car l'être humain n'est pas encore achevé, il est conscient de son inachèvement et se trouve en mouvement permanent de développement, selon Freire (1974). Le « plus-être » est la vocation ontologique et historique des hommes, c'est la construction prometteuse de leur épanouissement, l'humanisation de l'homme. 
chose à apporter. La confiance résulte des trois éléments précédemment établis. L'espoir est la conviction que le changement est possible ; c'est la compréhension de différents points de vue et les possibilités qui en découlent pour le monde. L'espoir réveille la quête et le mouvement nécessaires à la transformation. La criticidade $^{15}$, qui signifie conscience ou esprit critique, est permise par un regard ouvert et plein d'espoir, alimenté par une expérience humaine diversifiée. Lorsque l'on pense que le changement est possible, il devient plus facile de développer une réflexion critique sur ce qui, au fond, est inadéquat. C'est en quelque sorte l'action de problématiser les expériences individuelles et la société, en mettant en question sa propre insertion dans le monde et les conditions que ce monde lui offre.

En analysant le travail bénévole des conteurs brésiliens de la Casa, nous trouvons des affinités et des contradictions avec l'exercice du dialogue tel que le propose Freire (1974). Tout d'abord, les conteurs mettent en valeur l'amour (plus précisément «l'amoureusité ») comme sentiment indispensable à la rencontre conteur-public. Voisin de l'idée d'amour, le « pouvoir amoureux » de la narration est célébré par les narrateurs. La considération que les narrateurs manifestent envers des individus qui ne trouvent pas leur place dans la société hypermoderne en raison de leurs conditions physiques ou mentales nous semble être une expression de respect, voire une forme d'amour. L'effort des jeunes pour réussir à répondre d'une façon possible à la présence du conteur est, aussi, reconnaissance, donc amour/amoureusité.

Bien que la notion de « foi » des narrateurs soit différent de celui de Freire, en pratique ils partagent la même croyance dans le potentiel de l'autre et de la société, ainsi que dans le «plus-être ». Raconter pour changer son esprit et permettre, en même temps, à l'autre d'y arriver est, dans une certaine mesure, un exercice de foi. La foi en la transformation de l'autre est un point fondamental de l'action $\mathrm{du}$ 《récit social » car les narrateurs font confiance au développement de ces jeunes, parfois dans un état grave de paralysie et d'absence. Le potentiel de ce public particulier pourrait très bien échapper aux narrateurs, mais bien au contraire les changements comportementaux et physiques nourrissent et renforcent leur conviction.

Les narrateurs font preuve d'humilité : ce sont les histoires qui sont à leurs yeux les plus importantes, pas les conteurs, afin que la narration devienne source de transformations. L'humilité place les narrateurs dans une position de collaborateurs du processus narratif, qui aux côtés du conte et du public,

15. Caractéristique de celui qui est critique ; capacité d'analyser et de critiquer. 
Renouveau du conte et action sociale au Brésil : des conteurs bénévoles en dialogue avec leur public en situation de handicap

construisent une expérience riche de subjectivité et capable de produire des effets observables. « À ce point de rencontre, il n'y a ni ignorants, ni savants absolus : il y a des hommes [et femmes] qui, ensemble, essaient de savoir d'avantage » (Freire, 1974, p. 75). Ces trois éléments établis, se révèle une certaine confiance, toujours objet du discours des narrateurs. Elle se réalise par la croyance à la sincérité et à la disponibilité des participants des cercles.

La notion d'espoir est aussi indispensable au dialogue selon Freire. Pour les conteurs, l'espoir a un sens important, car la quête de la transformation du monde et des relations humaines est en soi une preuve de l'espoir que la réalité brésilienne et la condition humaine peuvent être améliorées. Pour l'auteur :

L'espérance n'incite pas à se croiser les bras et attendre. L'espérance me pousse à lutter, et si je lutte, c'est parce que je suis dans l'espérance. Si le dialogue est la rencontre des hommes en vue d'un « plus-être », il ne peut pas s'établir dans le désespoir. Quand ceux qui se parlent n'ont pas d'espérance, il ne peut y avoir de dialogue. Leur rencontre est vide et stérile. Elle est bureaucratique et sans intérêt. (1974, p.76)

Finalement, les conteurs agissent aussi dans l'expectative de développer leurs consciences critiques, la criticidade de Freire (1974), et d'aider les auditeurs à faire de même. La sortie d'un état d'apathie des jeunes ayant des handicaps, comme nous en avons été témoin, est un exemple de prise de conscience, bien qu'apparemment simple. Les conteurs semblent faire confiance à la capacité de réflexion, d'action et de transformation de ce public lors de la rencontre autour des contes.

Dans Pedagogia da esperança, une métathéorie ultérieure de Freire (2011), viennent s'ajouter trois conditions supplémentaires à l'exercice du dialogue : la démocratie, le silence et l'unité de la diversité. Il défend l'idée que la démocratie est l'espace égalitaire où la transformation peut germer. Le silence dans les relations humaines ne doit être ni absence ni indifférence, car il contribuerait alors à la « culture du silence ${ }^{16} \gg$ des opprimés. Le silence est donc écoute de l'autre et réflexion sur le dialogue lui-même. Plutôt que de valoriser les différences pour construire des savoirs pluriels, l'unité de la diversité est la capacité humaine, par le dialogue, de comprendre des réalités différentes, de reconnaître et de valoriser les similitudes. C'est le « ciment » des groupes en quête de transformation sociale.

16. Selon Freire $(1974,1981)$, dans la « culture du silence » la foule est muette, parce que l'individu n'a pas le droit de faire partie intégrante de la transformation sociale. 
Quand nous mettons en parallèle les trois éléments de la métathéorie freirienne et la pratique des conteurs observés, nous trouvons aussi des proximités et des écarts. Dans la mesure où le cercle est une réunion horizontale à laquelle tout le monde peut participer, raconter et s'exprimer de différentes manières, nous pouvons considérer qu'il s'agit de « démocratie ». Les conteurs veulent produire dans le cercle un espace démocratique dans lequel toutes les personnes concernées peuvent s'exprimer en donnant leurs avis. La figure du conteur, expression de pouvoir, peut être considérée comme incitatrice de ce dialogue. Cependant, s'il se place debout devant son public, tandis que les auditeurs sont assis, la dimension de « pouvoir » dans le cercle reste concentrée sur lui. Alors, la possibilité d'une rencontre horizontale devient une expérience verticale, où le conteur est au sommet comme référence de pouvoir et de savoir.

Dans un cercle d'inclination démocratique, tous auraient à contribuer, tous seraient plutôt assis, partageant la parole et le silence. Le silence traduit un état d'esprit et peut être considéré comme action et réaction, même s'il ne provoque pas d'effets clairement visibles. Il est particulièrement présent dans un cercle comme moment de raisonnement suivi d'une réaction. Bien que l'état de santé des jeunes ayant des handicaps prive la majorité de la parole, ils ne restent pas tout le temps dans le silence. Ils s'expriment d'autres manières, par le corps, des sons ou des attitudes, faisant preuve d'une attention, conscience et d'une présence élargies pendant un cercle.

Du point de vue kinésique (Birdwhistell, 1968), nous pouvons affirmer que les réactions des jeunes varient en intensité, en amplitude et en rapidité dans une perspective de communication polysémique, avec des résonances affectives. Les jeunes en situation de handicap observés expriment un langage non verbal et paraverbal (ex. la respiration) que nous pourrions, par exemple, mettre en relation avec les gestes décrits par Barrier (2019), tels que les gestes rythmiques, déictiques et pantomimiques, ainsi que les expressions faciales. Bien qu'ils envisagent de systématiser un éventuel code de communication non verbale/paraverbale, pour rechercher le sens et la logique de ces expressions, les narrateurs ne se sont pas encore engagés dans une cartographie des réactions de ces jeunes. Il pourrait être intéressant - pour nous, mais aussi pour les conteurs dans leur volonté de mieux tenir compte des spécificités de leur public - d’identifier, par exemple, les kinés, les kinèmes, les kinémorphes et les kinémorphèmes (Birdwhistell, 1968) de cette communication propre aux auditeurs en question. Dans cette perspective, il serait possible de savoir si ces réactions toujours présentes ont un objectif spécifique, si elles sont préconscientes, ou si elles ne sont que la simple expression des sentiments - tels que la quête d'une identification au conteur ou la volonté de participer au cercle. 
Renouveau du conte et action sociale au Brésil : des conteurs bénévoles en dialogue avec leur public en situation de handicap

Chaque public est unique de la même façon que les conteurs, issus de différentes couches sociales, professions et expériences de vie... D'un point d'intersection se produit la rencontre, moment ouvert pour les raisonnements à partir de contes. L'unité de la diversité est la « colle » qui les lie.

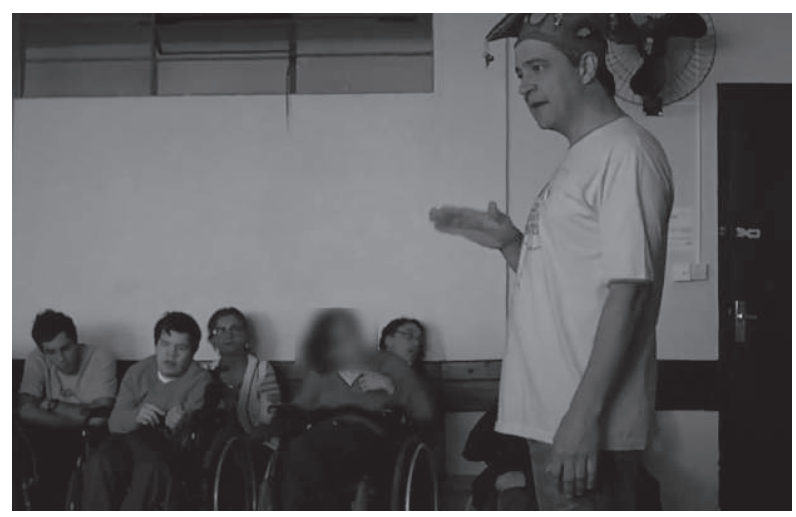

Photo 7

Le conteur Reinaldo, menuisier artisan, racontant à de jeunes brésiliens en situation de handicap.

(C) Rossane Lemos, 2014

Devant ces aspects d'intersection et d'écart entre la pratique des conteurs de la Casa et les théories de Freire à propos du dialogue, nous pouvons penser à une relation dialogique partielle entre eux. Les conteurs ne réalisent pas cette dimension théorique de la narration dialogique, mais ils sont curieux à propos des nouveaux regards qui pourraient enrichir leur « récit social ».

\section{Conclusion}

Nous avons vu qu'il est possible de faire le lien entre les éléments qui sont à la base du « récit social » des bénévoles de la Casa et les éléments indispensables à l'installation du dialogue selon Freire. Nous pouvons en conclure que les conteurs bénévoles sont en quête de dialogue, malgré les contradictions observées dans leur pratique. La notion de « dialogue » suppose, en général, la parole. Néanmoins, il parait évident que tant les narrateurs que les jeunes atteints de paralysie cérébrale s'engagent dans une expérience de dialogue. Ce public en situation de handicap, nonobstant d'importants troubles de la parole, établit un lien avec le narrateur et participe de la rencontre. Les manifestations s'observent non seulement lorsqu'un auditeur bégaie des extraits de l'histoire, mais également quand un 
enfant quitte l'état d'apathie ou de paralysie dans lequel il était, quand son corps parle pour lui et quand ses postures et ses mouvements se trouvent modifiés pendant la narration. Pour leur part, les conteurs font des efforts pour développer leur activité bénévole dans des références d'amoureusité, foi, humilité, etc. qu'ils possèdent. Par l'intermédiaire des contes, les narrateurs et les auditeurs élargissent leur répertoire d'expériences et essaient de développer leurs consciences dans une perspective de construction du «plus-être ».

Cependant, quand le narrateur fait le choix du répertoire adressé à son public de façon verticale, d'autorité, il se sépare d'une expérience complète de dialogue et d'une relation dialogique dans son principe. La construction du répertoire des histoires ne s'aligne pas sur cette perspective dialogique, quand il est choisi « pour » et non « avec » le public.

Les éléments qui confirment l'existence d'une relation partielle de dialogue entre les narrateurs de la Casa et leurs publics en situation de handicap sont des invitations à d'autres analyses. L'ensemble des situations observées présentées dans cet article peut motiver des personnes captivées à perfectionner l'expérience du dialogue dans des cercles d'histoires, en vue de mettre en place des nouvelles initiatives susceptibles de produire des transformations individuelles et collectives.

\section{Bibliographie}

Adotti Moacir, 1996, Paulo Freire: uma biobibliografia [Paulo Freire : une bibliographie], Ed. Cortez, São Paulo, 765 p.

BARrier Guy, 2019, La Communication non verbale : comprendre les gestes, perception et signification, Éditeur ESF, Montrouge, 200 p.

Birdwhistell Ray L. \& Lacoste Michèle, 1968, « L'analyse kinésique » in Langages, $\mathrm{n}^{\circ} 10$, p. 101-106.

Busatto Cléo, 2007, A Arte de Contar Histórias no Século XXI: Tradição e Ciberespaço [L'art de raconter des histoires au $\mathrm{XXI}^{\mathrm{e}}$ siècle : tradition et cyberespace], $2^{\mathrm{e}}$ éd., Ed. Vozes, Petrópolis, 152 p.

Calame-Griaule Geneviève (dir.), 1991, Le renouveau du conte, Éditions du CNRS, Paris, 449 p. 
Renouveau du conte et action sociale au Brésil : des conteurs bénévoles en dialogue avec leur public en situation de handicap

Campbell Charles Grimshaw, 1952, The Story of King Hamed ben Bathara, and of the Fearless Girl in BENN Ernest, From town and tribe, London, 217 p.

Campbell Joseph, 1997, O herói de mil faces, $14^{\mathrm{e}}$ éd., tit. orig. The hero with a thousand faces, Trad. Adail Sobral, São Paulo, Cultrix.

Dufour Michel, 1993, Allégories pour guérir et grandir, Psy Populaire/JCL, Paris, 223 p.

Freire Paulo, 1974, Pédagogie des opprimés, Librairie François Maspero, Paris, $205 \mathrm{p}$.

Freire Paulo, 1981, Ação cultural para a liberdade [L'action culturelle pour la liberté], $5^{\mathrm{c}}$ éd., Ed. Paz e Terra, Rio de Janeiro, 149 p.

Freire Paulo, 2011, Pedagogia da esperança - Um Reencontro com a Pedagogia do Oprimido [La pédagogie de l'espoir - une rencontre avec la pédagogie de l’opprimé], $17^{\mathrm{e} e ́ d ., ~ R i o ~ d e ~ J a n e i r o, ~ E d . ~ P a z ~ e ~ T e r r a, ~} 336$ p.

Freire Paulo, 2016, Pedagogia do oprimido [Pédagogie de l'opprimé], 60éd., Ed. Paz e Terra, Rio de Janeiro, 205 p.

Hernandez Soazig, 2006, Le Monde du conte - contribution à une sociologie de l'oralité, Éd. L'Harmattan, Paris, 317 p.

Holanda Sérgio Buarque (de), 1995, Raizes do Brasil [Les racines du Brésil], $26^{\mathrm{e} e}$ éd., Companhia das Letras, São Paulo, 113 p.

Jung Carl Gustav, 2000, Os arquétipos e o inconsciente coletivo [Les archétypes et l'inconscient collectif], Trad. Appy Maria et DA Silva Dora, Ed. Vozes, Petrópolis, $447 \mathrm{p}$.

KRISTEVA Julia, 1968, « Le geste, pratique ou communication ? » in Langages, $\mathrm{n}^{\circ} 10$, p. $48-64$.

Lanz Rudolf, 1997, Noçôes básicas de Antroposofia [Notions de base en anthroposophie], 4e éd., Livraria Antroposófica, São Paulo, 98 p. 
Lipovetsky Gilles \& Charles Sébastien, 2004, Des temps hypermodernes, Grasset, Paris, 196 p.

Morin Edgard, 1981, « Où va le monde ? » in Pour sortir du $X X^{e}$ siècle, Fernand Nathan, Paris, $384 \mathrm{p}$.

Morin Edgar, 1997, Amour, poésie et sagesse, Éd. du Seuil, Paris, 96 p.

Urbano Hudinilson, 2000, Oralidade na literatura: o caso Rubem Fonseca [Oralité en littérature : le cas de Rubem Fonseca], Cortez, São Paulo, 288 p.

VAlière Michel, 2006, Le Conte populaire - approche socio-anthropologique, Armand Colin, Paris 208 p.

Velasco Cristiane, 2018, Histórias de boca: o conto tradicional na educação infantil [Histoires de bouche : le conte traditionnel à l'école maternelle], Panda Books, São Paulo, 225 p.

Résumé : Les conteurs bénévoles de l'association « Casa do Contador de Histórias » de Curitiba (Brésil) étudiés dans cet article s'adressent à des publics en situation sociale précaire, comme les enfants et les adolescents atteints de paralysie cérébrale. Plus que la performance artistique, les narrateurs mettent en évidence dans leur pratique l'engagement associatif via la narration orale et le « pouvoir transformateur » des contes. Ils cherchent ainsi à contribuer au développement de la société brésilienne et à améliorer les relations humaines afin de renforcer l'esprit de groupe tout en développant la conscience des individualités. Après avoir présenté le contexte de narration bien particulier qui nous intéresse ici, nous verrons comment les pratiques narratives mettent en œuvre ce que nous proposons d'appeler un « récit social » afin de conter face à un public en situation de handicap, ce qui nous conduira à nous interroger sur la possibilité de dialogue qu'offre le type de narration proposé par les conteurs de la Casa à ces jeunes.

Mots-clefs : dialogue, conteur d'histoires, engagement associatif, bénévolat, récit, handicapés, Brésil, Paulo Freire 
Renouveau du conte et action sociale au Brésil : des conteurs bénévoles en dialogue avec leur public en situation de handicap

\section{Revival of Storytelling and Social Action in Brazil: Volunteer Storytellers in Dialogue with their Disabled Public}

Abstract: The volunteers storytellers of the "Casa do Contador de Historias" (House of Storyteller) association in Curitiba (Brazil) studied in this article target people in precarious social situations, such as children and adolescents with cerebral paralysis. More than artistic performance, storytellers highlight in their practice the voluntary-associative engagement through oral narration and the "transforming power" of tales. They aim to contribute to the development of Brazilian society and improve human relations, strengthening group spirit while developing an awareness of their individuality. After presenting the very particular narrative context that interests us here, we will see how narrative practices implement what we propose to call a "social narrative" in order to narrate stories to an audience of people with significant motor and mental disabilities. This will lead us to question the possibility of dialogue offered by the type of narrative proposed by the storytellers of the Casa to these young people.

Keywords: dialogue, storytellers, community engagement, volunteering, storytelling, disabled people, Brazil, Paulo Freire

\section{Renovação do conto e ação social no Brasil: os contadores voluntários brasileiros em diálogo com o público com paralisia cerebral}

Os contadores de histórias voluntários da associação "Casa do Contador de Histórias" em Curitiba (Brasil) estudados neste artigo se dirigem a pessoas em situação social precária, como crianças e adolescentes com paralisia cerebral. Mais do que a performance artistica, os narradores destacam em sua prática o engajamento associativo por meio da narração oral e o "poder transformador" dos contos. Eles buscam contribuir para o desenvolvimento da sociedade brasileira e melhorar as relaçôes humanas, a fim de fortalecer o espirito de grupo e desenvolver a consciência das individualidades. Depois de apresentar o contexto narrativo muito particular que nos interessa aqui, veremos como as práticas narrativas implementam o que propomos chamar de "narrativa social" a fim de narrar histórias para um público de portadores de necessidades especiais. Isto nos levará a questionar a possibilidade de diálogo oferecido pelo tipo de narrativa proposto pelos narradores da Casa a esses jovens. 
Palavras chave: diálogo, contadores de histórias, engajamento associativo, voluntariado, narração, pessoa com deficiência, Brasil, Paulo Freire

Rossane Lemos, doctorante en anthropologie, prépare une thèse sur les conteurs bénévoles au sud du Brésil, sous la direction de Cécile Leguy, à l'Université Sorbonne Nouvelle - Paris 3. 